\title{
STABILITY ANALYSIS AND DYNAMIC BEHAVIOUR OF A FLEXIBLE ASYMMETRIC ROTOR SUPPORTED BY ACTIVE MAGNETIC BEARINGS
}

\author{
Molka Attia Hili, Slim Bouaziz, Mohamed Haddar \\ Laboratory of Mechanical Modeling and Production (LA2MP), National School of Engineers of Sfax (ENIS), \\ University of Sfax, Tunisia; e-mail: Molka.hili79@gmail.com
}

The objective of this work is to show the influence of dynamic characteristics of Active Magnetic Bearings (AMBs) on the stability and dynamic response of an asymmetric and unbalanced rotor. Indeed, AMBs have been successfully applied in several industrial machinery facilities. Their main advantages are the contactless working principle, frictionless suspension and operation in very high speeds. Firstly, the AMBs dynamic support parameters have been obtained through electromagnetic theory. Then, a generalized system equations of motion have been derived using the finite element method. The motion of a rotor the shaft cross-section of which is asymmetric is generally governed by ordinary differential equations with periodic coefficients. Floquet's theory is used to investigate the stability of this system of equations. Finally, numerical simulation results are presented and discussed.

Keywords: asymmetric rotor, finite element, Floquet's theory, dynamic coefficients, stability

\section{Introduction}

A spinning system serves as a model for many rotating machinery elements. It is generally composed of a flexible shaft on which a flexible or rigid disk is mounted and supported by bearings. Bearings have a considerable effect on the dynamic behaviour of such systems. Recently, AMBs are increasingly used, especially in machines operating at very high rotational speeds, because of their many advantages (no lubrication, very long life, supporting hard environments, precise control, low power use and high-speed operating) compared to rolling elements, hydrodynamic or elasto-hydrodynamic bearings.

On the other hand, the presence of defects is a major concern in rotating machinery; they generate some important loads and vibrations and also stability problems. Asymmetric cross section of the shaft is among commonly encountered defects, it is usually due to machining defects.

The study of rotating systems supported by AMBs bearings and analysis of machine faults has resulted in an extensive body of publications.

Lei and Palazzolo (2008) presented an approach for the analysis and design of magnetic suspension systems with a large flexible rotor dynamic model including dynamics, control and simulation. Inayat-Hussain (2007) presented a numerical study to investigate the response of an unbalanced rigid rotor supported by AMBs. The mathematical model of the rotor-bearing system used in that study incorporated non-linearity arising from the electromagnetic force-coil and current-air gap relationship, and the effects of geometrical cross-coupling. The response of the rotor was observed to exhibit a rich variety of dynamical behaviour including synchronous, sub-synchronous, quasi-periodic and chaotic vibrations. Inagaki et al. (1980) studied a multi-disk fully asymmetric rotor with longitudinal variation of the shaft cross section. The temporal equations of motion were obtained using the transfer matrix method. The unbalance response was deduced by the harmonic balance method. 
Oncescu et al. (2001) proposed modifications into a classical finite element procedure developed for rotors with symmetry to incorporate the effect of shaft asymmetry and used Floquet's theory to investigate the stability of a general system of differential equations with periodic coefficients.

Recently, Inayat-Hussain (2010) studied the dynamics of a rigid rotor supported by load-sharing between magnetic and auxiliary bearings for a range of realistic design and operating parameters. Numerical results of that work show that the unbalance parameter is the main factor that influences the dynamics of the rotor-bearing system. It was also shown that the non-synchronous vibration response amplitude of the rotor with a relatively small unbalance magnitude can be reduced by decreasing the magnitude of the friction coefficient. Tsai et al. (2011) developed a wavelet transform algorithm to identify magnetic damping and stiffness coefficients of the driving rod with a set of 4-pole AMBs. This work further revealed that the identified second-order damping coefficient is negative for a specific rod displacement and speed. The dynamics of the rotor-AMBs system in the axial direction is unstable. Bouaziz et al. (2011) investigated the dynamic response of a rigid misaligned rotor mounted in two identical AMBs. Three simplified models of current biased radial AMBs were presented, where four, six and eight electromagnets were powered by a bias current and the respective control current. Results of that work show that angular misalignment is such that the $2 \times$ and $4 \times$ running speed components are predominant in spectra of vibration. Their magnitudes vary with the number of magnets in the bearing. Bouaziz et al. (2016), proposed a dynamical analysis of a high speed AMB spindle in the peripheral milling process. The time history of the response, orbit, FFT diagram at the tool-tip center and the bearings dynamic coefficients were plotted to analyze dynamic behavior of the spindle.

Most of the papers found in the literature concerning magnetic bearings are interested in the dynamic response of unbalanced or misaligned rotors. The shaft is generally considered rigid or massless.

On the other hand, the papers dealing with asymmetric shafts, consider that the shaft is supported by two identical elastic bearings. The coefficients of stiffness and damping are given arbitrarily. Stability study is very limited.

In this paper, dynamic characteristics of Active Magnetic Bearings (AMBs) is first be determined. Then a model of an asymmetric rotor supported by two magnetic bearings is presented using the finite element procedure. A stability analysis will be conducted while showing the influence of various parameters of the bearings on stability areas. In the same way, the dynamic response of the asymmetric shaft will be calculated and analyzed.

\section{Bearing modelling}

The electromagnetic bearing studied is formed by four electromagnets $(n=4)$ placed in the bearing around the rotor and producing an attractive force (Fig. 1).

Using the electromagnetic theory, the electromagnetic resultant forces produced by every pair of the electromagnets in $x$ and $y$ directions are expressed as (Inayat-Hussain, 2010)

$$
F_{x}=\lambda\left[\left(\frac{I_{0}-i_{x}}{C_{0}-u_{x}}\right)^{2}-\left(\frac{I_{0}+i_{x}}{C_{0}+u_{x}}\right)^{2}\right] \quad F_{y}=\lambda\left[\left(\frac{I_{0}-i_{0}-i_{y}}{C_{0}-u_{y}}\right)^{2}-\left(\frac{I_{0}+i_{0}+i_{y}}{C_{0}+u_{y}}\right)^{2}\right]
$$

where $C_{0}$ is the nominal air gap, $i_{0}$ is the bias current (to produce neutralizing force due to weight of the rotor), $I_{0}$ is the steady state current in the coil, $u_{x}$ and $u_{y}$ are respectively the shaft displacements in the $x$ and $y$ directions, $\lambda$ is the global magnetic permeability expressed as

$$
\lambda=\frac{\mu_{0} A N^{2}}{4} \cos \theta
$$




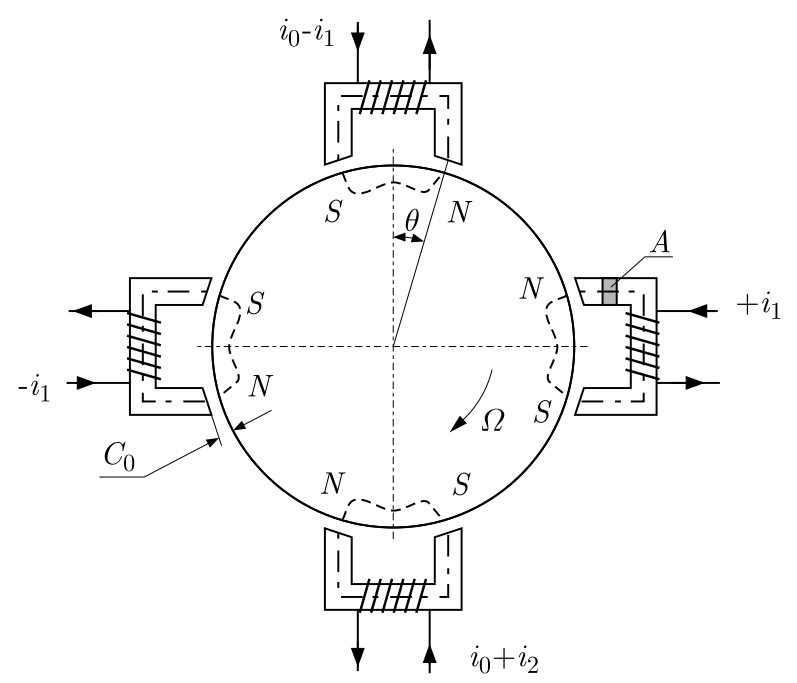

Fig. 1. Electromagnetic bearing

where $A, \mu_{0}, \theta$ and $N$ represent, respectively, cross section area of an electromagnet, permeability of vacuum, half angle between the poles of the electromagnet and the number of windings in the coil, and $i_{j}(j=x, y)$ represents the control current expressed using a proportional-differential (PD) controller as

$$
i_{j}=k_{p} u_{j}+k_{d} \dot{u}_{j} \quad j=x, y
$$

where $\dot{u}_{j}$ is shaft velocity in the $j$ direction, $k_{p}$ is the proportional gain and $k_{d}$ is the differential gain.

By replacing (2.3) respectively in (2.1), we obtain

$$
\begin{aligned}
& F_{x}=a\left[\left(\frac{1-\frac{k_{p} u_{x}}{I_{0}}-\frac{k_{d} \dot{u}_{x}}{I_{0}}}{1-\frac{u_{x}}{C_{0}}}\right)^{2}-\left(\frac{1+\frac{k_{p} u_{x}}{I_{0}}+\frac{k_{d} \dot{u}_{x}}{I_{0}}}{1+\frac{u_{x}}{C_{0}}}\right)^{2}\right] \\
& F_{y}=a\left[\left(\frac{1-\frac{i_{0}}{I_{0}}-\frac{k_{p} u_{y}}{I_{0}}-\frac{k_{d} \dot{u}_{y}}{I_{0}}}{1-\frac{u_{y}}{C_{0}}}\right)^{2}-\left(\frac{1+\frac{i_{0}}{I_{0}}+\frac{k_{p} u_{y}}{I_{0}}+\frac{k_{d} \dot{u}_{y}}{I_{0}}}{1+\frac{u_{y}}{C_{0}}}\right)^{2}\right]
\end{aligned}
$$

where $a=\lambda I_{0}^{2} C_{0}^{2}$.

Electromagnetic forces that depend on the shaft centre displacement and velocity are linearized (first order) around the equilibrium position (Bouaziz et al., 2016). This will provide the classic model of a bearing with four stiffness and damping coefficients (Fig. 2)

$$
\left\{\begin{array}{l}
f_{x} \\
f_{y}
\end{array}\right\}=-\mathbf{K}_{B}\left\{\begin{array}{l}
u_{x} \\
u_{y}
\end{array}\right\}-\mathbf{C}_{B}\left\{\begin{array}{l}
\dot{u}_{x} \\
\dot{u}_{y}
\end{array}\right\}
$$

where $\mathbf{K}_{B}$ is the bearing stiffness matrix expressed as

$$
\mathbf{K}_{B}=\left[\begin{array}{ll}
K_{x x} & K_{x y} \\
K_{y x} & K_{y y}
\end{array}\right]=-\left[\begin{array}{cc}
\left(\frac{\partial F_{x}}{\partial u_{x}}\right)_{0} & 0 \\
0 & \left(\frac{\partial F_{y}}{\partial u_{y}}\right)_{0}
\end{array}\right]
$$

and $\mathbf{C}_{B}$ is the bearing stiffness matrix expressed as

$$
\mathbf{C}_{B}=\left[\begin{array}{ll}
c_{x x} & c_{x y} \\
c_{y x} & c_{y y}
\end{array}\right]=-\left[\begin{array}{cc}
\left(\frac{\partial F_{x}}{\partial \dot{u}_{x}}\right)_{0} & 0 \\
0 & \left(\frac{\partial F_{y}}{\partial \dot{u}_{y}}\right)_{0}
\end{array}\right]
$$


In this model, the stiffness and damping cross-coefficients of the bearings are neglected. Numerical differentiation method is selected for determination of the dynamic coefficients. The partial derivatives are evaluated by the finite difference central method.

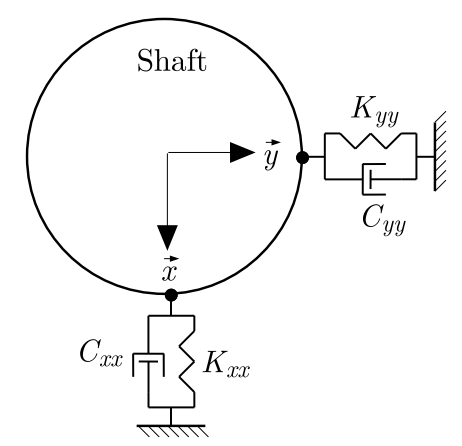

Fig. 2. Two DOF bearing model

\section{Equation of motion}

The mathematical model (Fig. 3) consists of a flexible asymmetric shaft, one rigid disk and two active magnetic bearings.

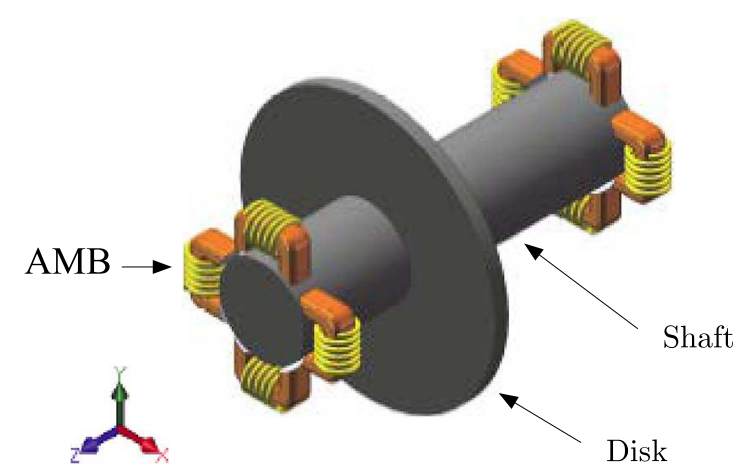

Fig. 3. Rotor bearing system with AMBs

The finite element procedure for rotors with the symmetric shaft is considered. Modifications are made to accommodate the effect of shaft asymmetry (Oncescu et al., 2001).

\subsection{Equation of motion of the shaft}

The shaft is considered to be flexible. It is characterized by its kinetic and deformation energies. Its motion results from transverse displacement $\left(u_{x}, u_{y}\right)$ and bending deformations $\left(\theta_{x}, \theta_{y}\right)$ in the $x$ - and $y$-planes (Fig. 4).

Because of the shaft asymmetry, the sectional moments of inertia $I_{x}$ and $I_{y}$ are not identical, consequently, the kinetic energy of the shaft can be represented by Oncescu et al. (2001)

$$
\begin{aligned}
T_{s} & \left.=\frac{\rho S}{2} \int_{0}^{L}\left(\dot{u}_{x}^{2}+\dot{u}_{y}^{2}+\dot{u}_{z}^{2}\right) d z+\frac{\rho I_{m}}{2} \int_{0}^{L}\left(\dot{\theta}_{x}^{2}+\dot{\theta}_{y}^{2}\right) d z+2 \rho I_{m} \Omega \int_{0}^{L} \dot{\theta}_{x} \theta_{y}\right) d z \\
& +\frac{\rho I_{d}}{2} \int_{0}^{L}\left(\dot{\theta}_{x}^{2}+\dot{\theta}_{y}^{2}\right) d z \cos (2 \Omega t)+\rho I_{d} \int_{0}^{L}\left(\dot{\theta}_{x} \theta_{y}\right) d z \sin (2 \Omega t)
\end{aligned}
$$




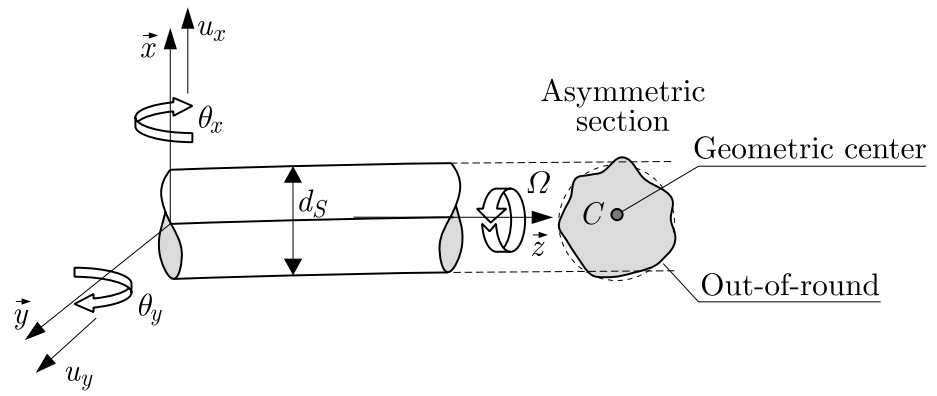

Fig. 4. Shaft modelling and corresponding DOF

where $\rho$ is material density, $I_{m}=\left(I_{x}+I_{y}\right) / 2, I_{d}=\left(I_{x}-I_{y}\right) / 2$ are respectively deviatory and mean area moments of inertia of the shaft cross-section $\left(I_{x}\right.$ and $I_{y}$ are the second moments of area about the principal axes $x$ and $y$ of the shaft).

Rayleigh's dissipation function of the disk is (Gosiewski, 2008)

$$
E_{d}=\frac{1}{2} \int_{0}^{L} c_{s}\left(\dot{u}_{x}^{2}+\dot{u}_{y}^{2}\right) d z+\frac{1}{2} \int_{0}^{L} c_{i}\left[\left(\dot{u}_{x}+\Omega u_{y}\right)^{2}+\left(\dot{u}_{y}-\Omega u_{x}\right)^{2}\right] d z
$$

where $c_{s}$ and $c_{i}$ are respectively coefficients of external and internal damping.

If shear deformations are neglected, the strain energy of the shaft is (Oncescu et al., 2001)

$$
\begin{aligned}
U= & \frac{1}{2} \int_{0}^{L} E I_{d}\left\{\left[\left(\frac{\partial^{2} u_{y}}{\partial z^{2}}\right)^{2}-\left(\frac{\partial^{2} u_{x}}{\partial z^{2}}\right)^{2}\right] \cos (2 \Omega t)-2 u_{x} u_{y} \sin (2 \Omega t)\right\} d z \\
& +\frac{1}{2} \int_{0}^{L} E I_{m}\left[\left(\frac{\partial^{2} u_{x}}{\partial z^{2}}\right)^{2}+\left(\frac{\partial^{2} u_{y}}{\partial z^{2}}\right)^{2}\right] d z
\end{aligned}
$$

where $E$ is Young's modulus.

The finite element used to discretize the shaft consists of two node beam elements where each node has four degrees of freedom: two lateral displacements and two bending rotation angles. Applying Lagrange's formalism to this system permits the development of the equations of motion of asymmetric shaft (Oncescu et al., 2001)

$$
\mathbf{M}_{s}(t) \ddot{\boldsymbol{\delta}}_{s}+\left(\Omega \mathbf{G}_{s}+\mathbf{C}_{s}\right) \dot{\boldsymbol{\delta}}_{s}+\mathbf{K}_{s}(t) \boldsymbol{\delta}_{s}=\mathbf{0}
$$

with

$$
\begin{aligned}
& \mathbf{M}_{s}(t)=\mathbf{M}_{s s}+\mathbf{M}_{d, c} \cos (2 \Omega t)+\mathbf{M}_{d, s} \sin (2 \Omega t) \\
& \mathbf{K}_{s}(t)=\mathbf{K}_{s s}+\mathbf{K}_{d, c} \cos (2 \Omega t)+\mathbf{K}_{d, s} \sin (2 \Omega t)
\end{aligned}
$$

where $\mathbf{M}_{s s}$ is the mass matrix of the symmetric shaft (Batoz and Gouri, 1990), $\mathbf{M}_{d, c}$ and $\mathbf{M}_{d, s}$ are mass matrices induced by the asymmetry of the shaft, $\mathbf{G}_{s}$ is the gyroscopic matrix of the shaft, $\mathbf{C}_{s}$ is the damping matrix, $\mathbf{K}_{s s}$ is the stiffness matrix of the symmetric shaft (Batoz and Gouri, 1990), $\mathbf{K}_{d, c}$ and $\mathbf{K}_{d, s}$ are stiffness matrices induced by the asymmetry of the shaft, $\boldsymbol{\delta}_{s}$ is the vector of shaft DOFs.

\subsection{Equation of motion of the disk}

The center of mass of the rigid disk coincides with the elastic center of the shaft cross-section. The nodal displacements vector of the disk in fixed co-ordinates is given by: $\boldsymbol{\delta}_{D}=\left\{u_{d, x}, u_{d, y}, \theta_{d, x}, \theta_{d, y}\right\}$. 
The kinetic energy of the disk, by considering the effect of the unbalance, is expressed as (Oncescu et al., 2001)

$$
T_{d}=\frac{1}{2} M_{d}\left(\dot{u}_{d, x}+\dot{u}_{d, y}^{2}\right)+\frac{1}{2} J\left(\dot{\theta}_{d, x}^{2}+\dot{\theta}_{d, y}^{2}\right)+2 J \dot{\theta}_{d, x} \theta_{d, y}+m_{u} d \Omega\left[\dot{u}_{d, x} \cos (\Omega t)+\dot{u}_{d, y} \sin (\Omega t)\right]
$$

where $M_{d}$ and $J$ are mass and moment of inertia of the disk, $\Omega$ is angular speed of the rotor, $m_{u}$ is unbalance mass (assumed to be small if compared with $M_{d}$ ), $d$ is the radius defining location of the unbalance.

Rayleigh's function of the disk energy dissipation is (Gosiewski, 2008)

$$
E_{d}=\frac{1}{2} c_{s}\left(\dot{u}_{d, x}^{2}+\dot{u}_{d, y}^{2}\right)+\frac{1}{2} c_{i}\left[\left(\dot{u}_{d, x}+\Omega u_{d, y}\right)^{2}+\left(\dot{u}_{d, y}-\Omega u_{d, x}\right)^{2}\right]
$$

where $c_{s}$ and $c_{i}$ are respectively the coefficients of external and internal damping.

The application of Lagrange's equations for the disk only gives

$$
\mathbf{M}_{D} H+\ddot{\boldsymbol{\delta}}\left(\Omega \mathbf{G}_{D}+\mathbf{C}_{D}\right) \dot{\boldsymbol{\delta}}_{D}+\mathbf{K}_{D} \boldsymbol{\delta}_{D}=\mathbf{F}_{u}(t)
$$

where $\mathbf{M}_{D}, \mathbf{G}_{D}, \mathbf{C}_{D}$ and $\mathbf{K}_{D}$ are respectively mass, gyroscopic, damping and stiffness matrices of the disk, $\mathbf{F}_{u}(t)$ is the unbalance vector.

\subsection{General equation of motion of the rotor}

By assembling the elementary matrices of shaft elements, disks and bearings (as expressed in Section 2), we obtain a system of $n$ second order differential equations and $n$ unknown functions, where $n$ is the number of DOFs of the rotor. The global equations of motion are

$$
\mathbf{M}(t) \ddot{\boldsymbol{\delta}}+(\mathbf{C}+\Omega \mathbf{G}) \dot{\boldsymbol{\delta}}+\mathbf{K}(t) \boldsymbol{\delta}=\mathbf{F}_{u}(t)
$$

where $\mathbf{M}(t)$ and $\mathbf{K}(t)$ are periodic matrices of period $T_{1}=\pi / \Omega$, for which the time dependency is due to shaft asymmetry, $\mathbf{C}$ is a constant matrix including damping effects of AM bearings, $\mathbf{G}$ is the gyroscopic matrix, $\mathbf{F}_{u}(t)$ - unbalance vector of period $T_{2}=2 \pi / \Omega$ and $\boldsymbol{\delta}$ is the vector of global DOFs.

The equations of motion are therefore parametric in nature, which usually causes a stability problem. Floquet's theory will be used to determine the zones of instability.

\section{Floquet's theory}

Floquet's method is a mathematical tool for solving parametric differential equations, such as (3.8). It involves computation of a transfer matrix over one period of motion (Dufour and Berlioz, 1998).

The study of stability of the steady state solution of system (3.8) can be reduced to study of stability of the trivial solution of the associated homogeneous system.

A state-space model for system (3.8) (with $\mathbf{F}_{u}(t)=\mathbf{0}$ ) has the form

$$
\dot{\mathbf{X}}=\mathbf{A}(t) \mathbf{X}
$$

where $\mathbf{A}(t)$ is a $m \times m(m=2 n)$ periodic matrix called the dynamic matrix of period $T$, and $\mathbf{X}=\{\delta, \dot{\delta}\}^{-1}$ is the state variable vector.

The transfer matrix $\boldsymbol{\Phi}\left(T, t_{0}\right)$ (Bauchau and Nikishkov, 2001) is by definition a matrix that relates the initial solution $\mathbf{X}\left(t_{0}\right)$ to the solution $\mathbf{X}(T)$ obtained at $t=T$, so

$$
\mathbf{X}(T)=\mathbf{\Phi}\left(T, t_{0}\right) \mathbf{X}\left(t_{0}\right)
$$


The period $T$ of the matrix $\mathbf{A}(t)$ is divided into $n$ intervals of equal length $h=T / n\left(t_{0}<t_{1}<\right.$ $\left.\ldots<t_{n-1}<t_{n}\right)$. Between the two solutions of (Eq. (3.7)) $\mathbf{X}\left(t_{i+1}\right)$ and $\mathbf{X}\left(t_{i}\right)$, there is a relation

$$
\mathbf{X}\left(t_{i+1}\right)=\mathbf{\Phi}\left(t_{i+1}, t_{i}\right) \mathbf{X}\left(t_{i}\right)
$$

where $\mathbf{\Phi}\left(t_{i+1}, t_{i}\right)$ is the elementary transfer matrix. We can easily notice that

$$
\boldsymbol{\Phi}\left(T, t_{i}\right)=\mathbf{\Phi}\left(T, t_{i+1}\right) \boldsymbol{\Phi}\left(t_{i+1}, t_{i}\right)
$$

The matrix $\boldsymbol{\Phi}\left(T, t_{0}\right)$ can be obtained by iterative calculation based on relation (4.4). We start with $\boldsymbol{\Phi}(T, T)=\mathbf{I}_{m}$ and gradually gets $\boldsymbol{\Phi}\left(T, t_{n-1}\right), \boldsymbol{\Phi}\left(T, t_{n-2}\right), \ldots, \boldsymbol{\Phi}\left(T, t_{0}\right)$.

To determine the elementary transfer matrix $\boldsymbol{\Phi}\left(t_{i+1}, t_{i}\right)$, several methods have been proposed. In this work, Newmark's method is used.

Furthermore, it is shown that the stability of the trivial solution of equation (3.7) is fully defined by the eigenvalues of the transfer matrix over one period $\boldsymbol{\Phi}\left(T, t_{0}\right)$, known as the characteristic multipliers of system. The trivial solution is asymptotically stable if the modulus of all $m$ eigenvalues is less than one, and is unstable if the modulus of at least one of the eigenvalues is greater than one.

\section{Numerical results}

The spinning system investigated in this paper is shown in Fig. 4. The disk is mounted on the shaft at $3 L / 4$. The system parameters are given in Table 1 .

Table 1. System parameters

\begin{tabular}{|l|c|c|}
\hline \multicolumn{1}{|c|}{ Parameter } & Symbol & Value \\
\hline \hline Permeability of vacuum & $\mu_{0}$ & $4 \pi \cdot 10^{-7} \mathrm{~Wb} / \mathrm{Am}$ \\
\hline Number of windings around core & $N$ & 300 \\
\hline Half angle between poles of electromagnet & $\theta$ & $22.5 \mathrm{deg}$ \\
\hline Bias current & $i_{0}$ & $0.5 \mathrm{~A}$ \\
\hline Differential gain & $k_{d}$ & $42.4 \mathrm{~A} \cdot \mathrm{s} / \mathrm{m}$ \\
\hline Proportional gain & $k_{p}$ & $14869 \mathrm{~A} / \mathrm{m}$ \\
\hline Rotor length & $L$ & $300 \mathrm{~mm}$ \\
\hline Shaft diameter & $d_{S}$ & $10 \mathrm{~mm}$ \\
\hline Disk diameter & $d_{D}$ & $100 \mathrm{~mm}$ \\
\hline Young's modulus & $E$ & $2.1 \cdot 10^{11} \mathrm{~Pa}$ \\
\hline Density & $\rho$ & $7.85 \mathrm{gm} / \mathrm{cm}^{3}$ \\
\hline Poisson's ratio & $\nu$ & 0.28 \\
\hline Unbalance mass & $M_{u}$ & $5 \mathrm{gm}$ \\
\hline Moment of inertia & $J$ & $0.005 \mathrm{Kg} \cdot \mathrm{m}^{2}$ \\
\hline Rotor running speed & $\Omega$ & - \\
\hline
\end{tabular}

Figures 5 and 6 show respectively the dependency of stiffness and damping coefficients of AMBs on the air gap $C_{0}$ between the stator and the rotor for different values of the steady state current in the coil $I_{0}$. Figures 7 and 8 show respectively the dependency of stiffness and damping coefficients of AMBs on the air gap $C_{0}$ between the stator and the rotor for different values of cross-sectional area of one electromagnet $A$.

$K_{x x}, K_{y y}, C_{x x}$ and $C_{y y}$ are strongly influenced by $C_{0}, I_{0}$ and $A$. Indeed, their values decrease considerably with $C_{0}$, but regularly increase respectively with $I_{0}$ and $A$. This observation was proven by Bouaziz et al. (2011) when they determine the dynamic coefficients of AMB with 
four, six and eight magnets. It is clear that the four coefficients become constant for large values of $C_{0}$ (from $C_{0}=2 \mathrm{~mm}$ and irrespective of $I_{0}$ and $A$ ). It should also be noted that the values of damping coefficients are significant compared to those frequently encountered in literature for modeling of elastic bearings.
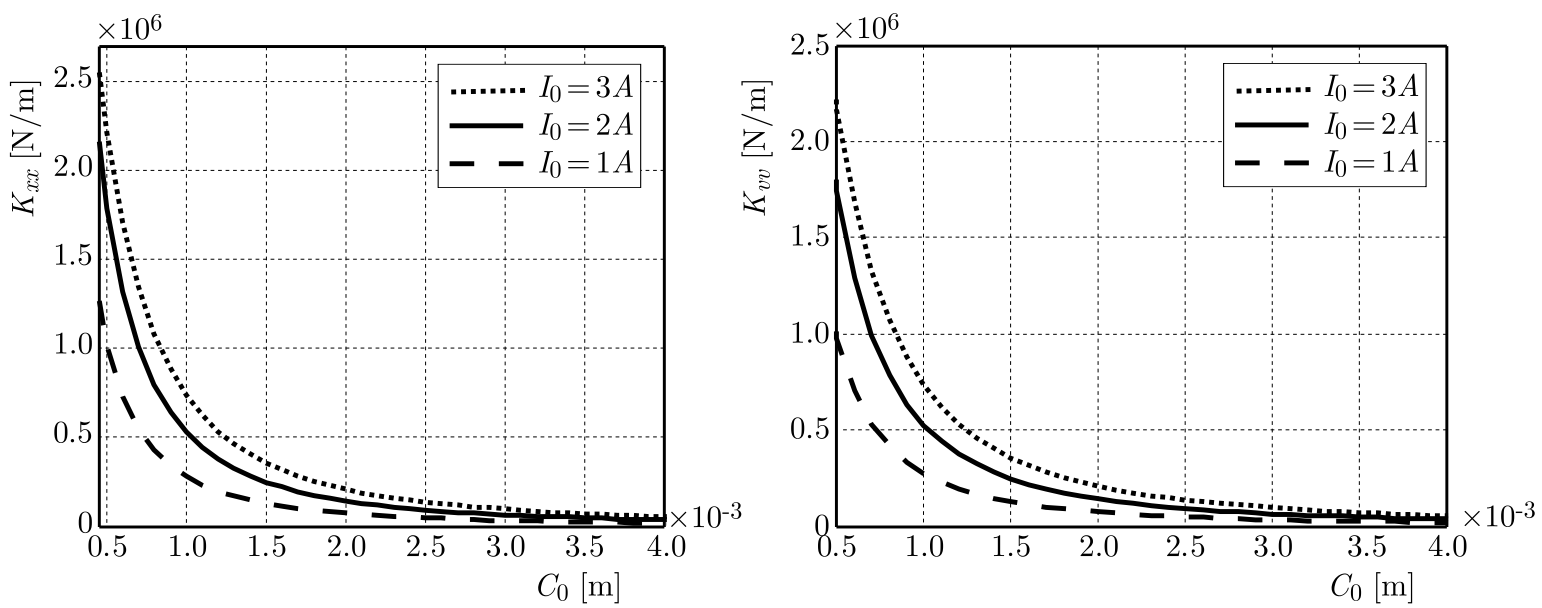

Fig. 5. Stiffness coefficients $\left(A=200 \mathrm{~mm}^{2}\right)$
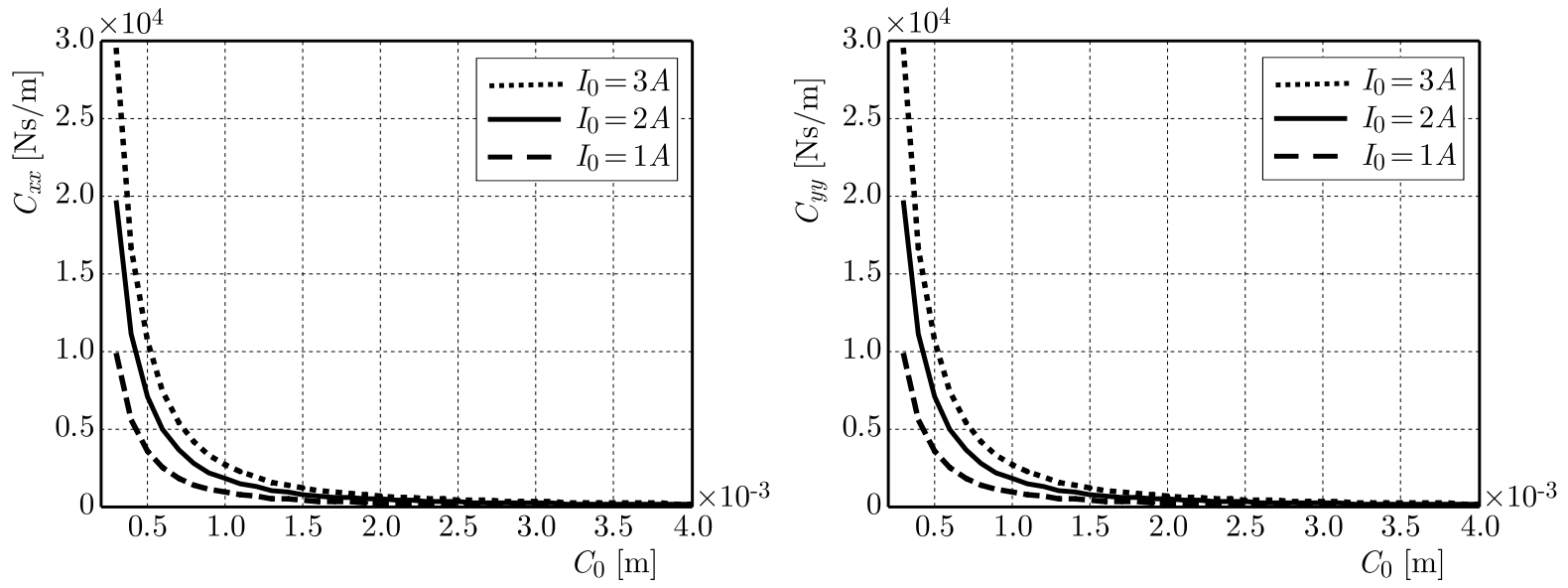

Fig. 6. Damping coefficients $\left(A=200 \mathrm{~mm}^{2}\right)$
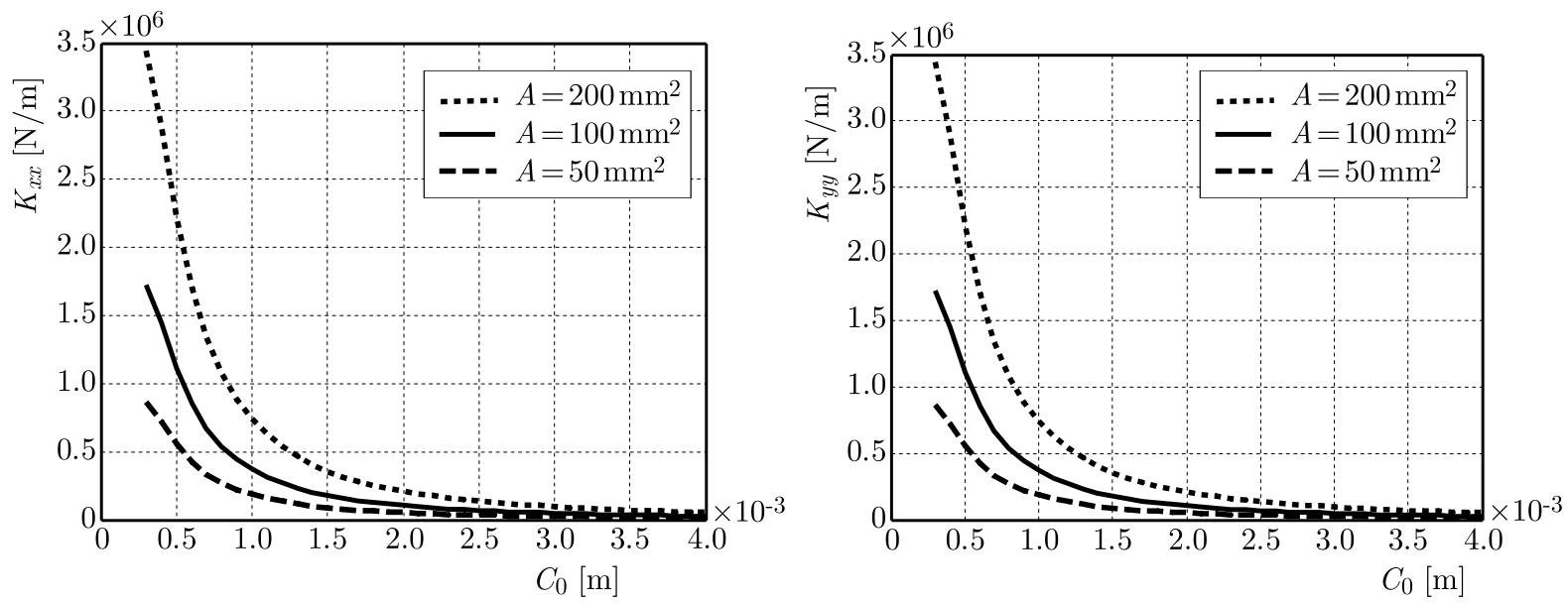

Fig. 7. Stiffness coefficients $\left(I_{0}=3 \mathrm{~A}\right)$ 

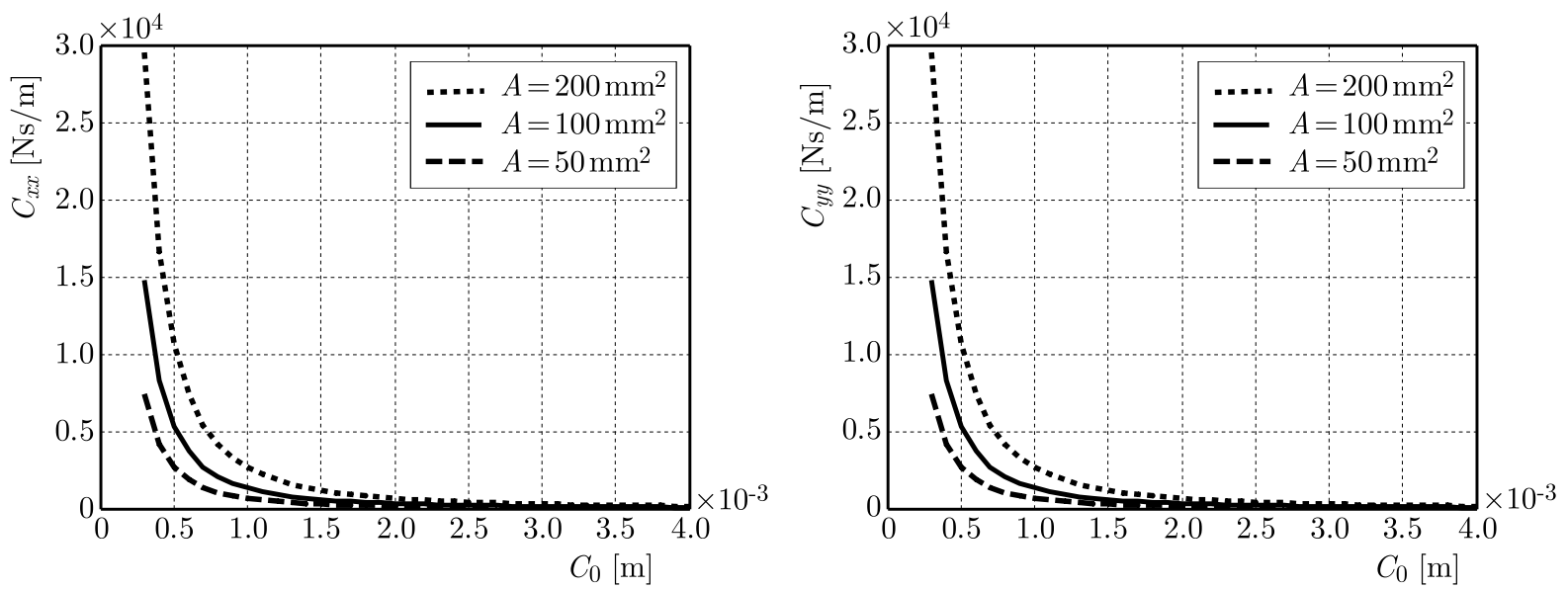

Fig. 8. Damping coefficients $\left(I_{0}=3 \mathrm{~A}\right)$

Since the coefficients of stiffness and damping are variable according to the parameters of the bearings, the stability of the studied asymmetric rotor varies as well. So try from the layout of different regions of stability to find the optimal parameters providing better system behavior (a minimum of regions of instability). The variable parameter is the factor of shaft asymmetry defined as the rate between the deviatory $\left(I_{d}\right)$ and the mean area moments of inertia of the shaft cross-section $\left(I_{m}\right): f s a=I_{d} / I_{m}$.

We are interested in the range of higher speeds because electromagnetic bearings are used in applications at high speeds.

Figure 9a shows regions of instability obtained for the studied system for factors of shaft asymmetry varying from 0 to 0.3 with increments by 0.05 . The results have been obtained by varying the rotational speed between 10000 and $80000 \mathrm{rpm}$, with increments by $100 \mathrm{rpm}$. The air gap $C_{0}$ is $0.5 \mathrm{~mm}$, the steady state current in the coil $I_{0}$ is $3 A$, and the effective cross-sectional area of one electromagnet $A$ is $200 \mathrm{~mm}^{2}$.
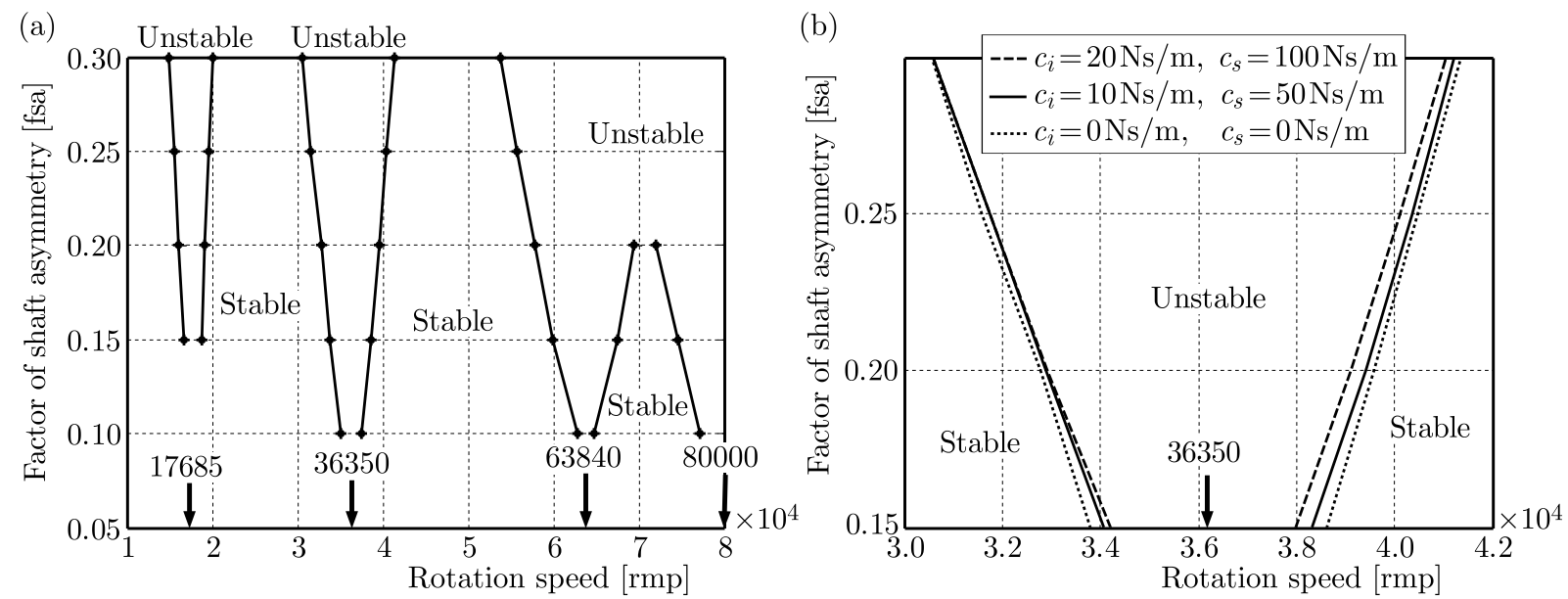

Fig. 9. Instability regions: (a) $C_{0}=0.5 \mathrm{~mm}, I_{0}=3, A=200 \mathrm{~mm}$, (b) [30000- $\left.40000 \mathrm{rpm}\right], C_{0}=0.5 \mathrm{~mm}$, $I_{0}=3, A=200 \mathrm{~mm}$

For the symmetric shaft, no instability interval has been identified around the four shaft critical speeds: 17685, 36350, 63840 and $80100 \mathrm{rpm}$ (according to the Campbell diagram). For the asymmetric shaft, four regions of instability appear for a factor of shaft asymmetry of 0.1 and for widths increasing with the shaft asymmetry. For a shaft asymmetry of 0.25 , there are 5 critical speeds delimiting the three regions of instability: 15492, 19639, 31600, 40475, and $55765 \mathrm{rpm}$. 
Figure $9 \mathrm{~b}$ shows the influence of internal and external damping on the instability region [30000-40000 rpm]. The width of the stability zone decreases substantially by introducing the internal and external damping. This behavior is also observed for the other two instability regions. These coefficients are generally negligible compared to those provided by AMBs and cannot in this case considerably influence the stability of the system studied.

Figure 10 depicts the regions of instability respectively obtained for three different values of $C_{0}: 0.5 \mathrm{~mm}, 1.5 \mathrm{~mm}$ and $3 \mathrm{~mm}$.

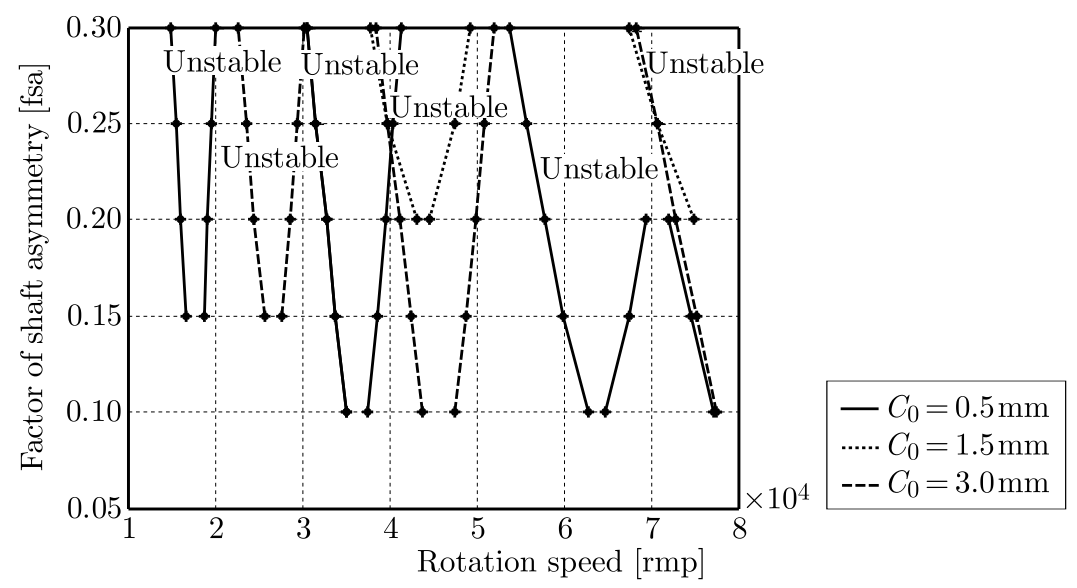

Fig. 10. Instability regions, $I_{0}=3, A=200 \mathrm{~mm}$

It is evident that the instability regions are highly dependent on the air gap $C_{0}$. Indeed, for a small value of $C_{0}\left(C_{0}=0.5 \mathrm{~mm}\right)$, the stiffness coefficients of AMBs are very important (Fig. 5). They increase the critical speed of the system and, therefore, the stability regions are also affected.

We note the presence of three regions of instability for $C_{0}=3 \mathrm{~mm}$ against two for $C_{0}=1.5 \mathrm{~mm}$.

The second region of instability begins from a factor of shaft asymmetry of 0.2 for $C_{0}=1.5 \mathrm{~mm}$, while it starts from a factor of shaft asymmetry of 0.1 for $C_{0}=3 \mathrm{~mm}$. In fact, for greater values of $C_{0}$, the stiffness coefficients of AMBs undergo a slight decrease. The positions of instability regions remain therefore almost unchanged, while their size and numbers increase with $C_{0}$. This last behavior is explained by a decrease in damping coefficients of the AMBs.
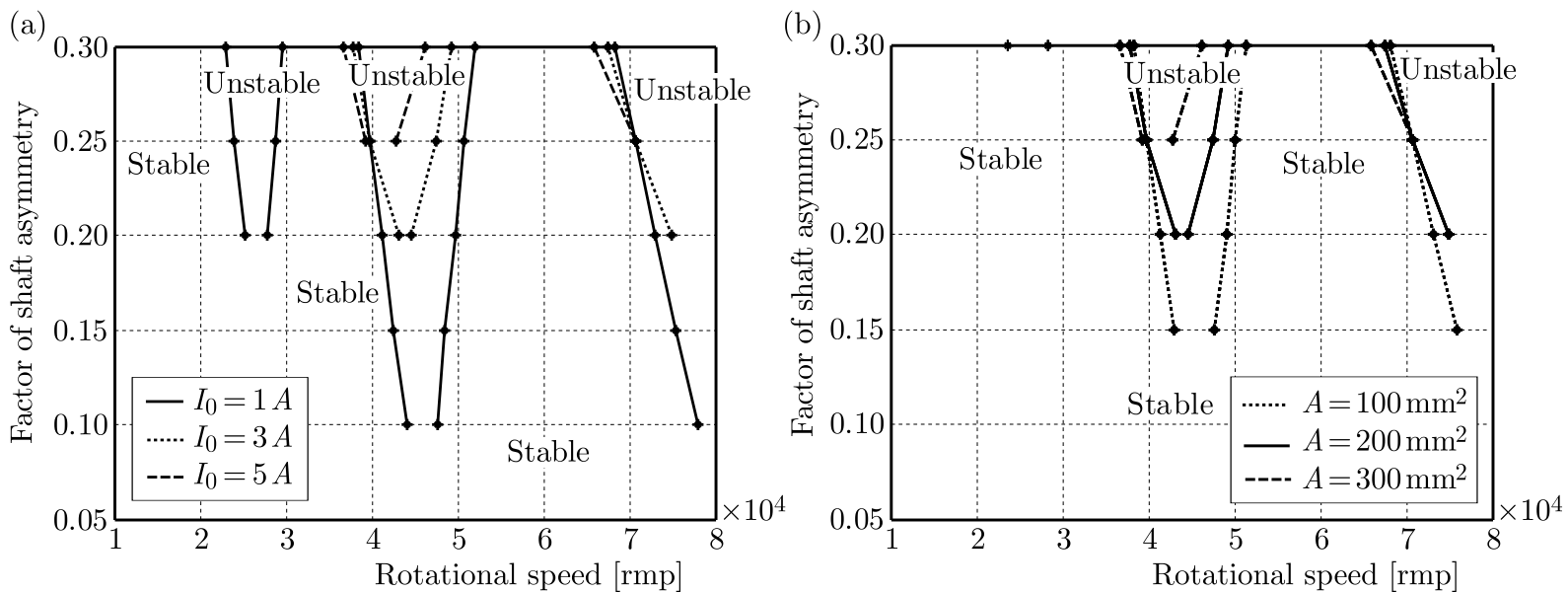

Fig. 11. Instability regions: (a) $C_{0}=1.5 \mathrm{~mm}, A=200 \mathrm{~mm}$, (b) $C_{0}=1.5 \mathrm{~mm}, I_{0}=3 \mathrm{~A}$

Figure 11a shows regions of instability obtained for three values of $I_{0}$. It is observed that regions of instability decreas with the increasing $I_{0}$. By increasing $I_{0}$, we can also remove a 
region of instability, which proves the importance of control. Figure $11 \mathrm{~b}$ presents the regions of instability obtained for three values of $A$. It is also observed that regions of instability decrease with the increasing $A$.

We can conclude that the stability is strongly linked with the dynamic coefficients of the bearings. A significant change of stiffness coefficients results in a change of the critical speed of the system and, consequently, a change in the positions of instability areas. At the same time, an increase in the damping coefficients, automatically leads to a decrease in the size of instability regions. $C_{0}$ and $I_{0}$ are the most influential parameters on the rotor stability. Based on this analysis, the parameters of AMBs that offer greater stability to the asymmetric rotor are: $C_{0}=1.5 \mathrm{~mm}, A=200 \mathrm{~mm}$ and $I_{0}=5 \mathrm{~A}$.

To better understand the instability phenomenon of asymmetric rotors, it is important to calculate and analyze the dynamic response. The dynamic response is observed at the disk-toshaft attachment. A spectral method is used to estimate the dynamic response (Attia Hili et al., 2006).

The frequency response along the $x$ direction (power spectral density) of the rotor in the frequency range of 0 to $250 \mathrm{~Hz}$ is shown in Fig. 12a. The factor of asymmetry is 0.25 when the shaft running speed is $420 \mathrm{rpm}$.

(a)

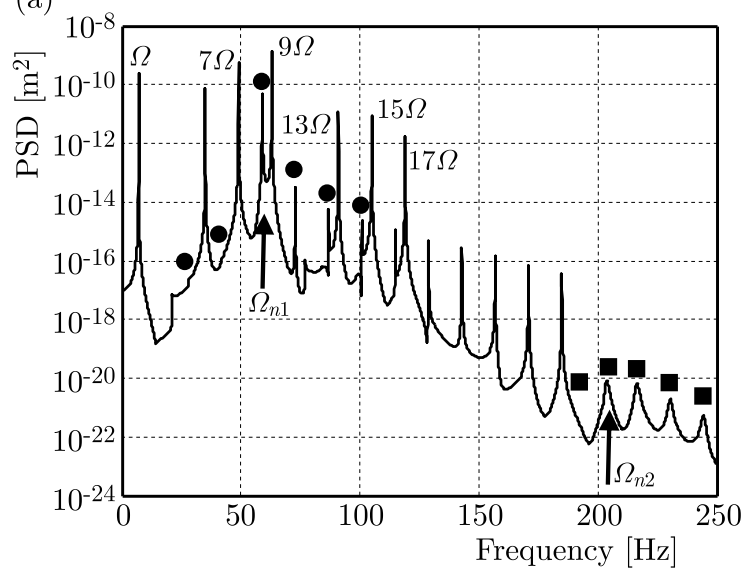

(b)

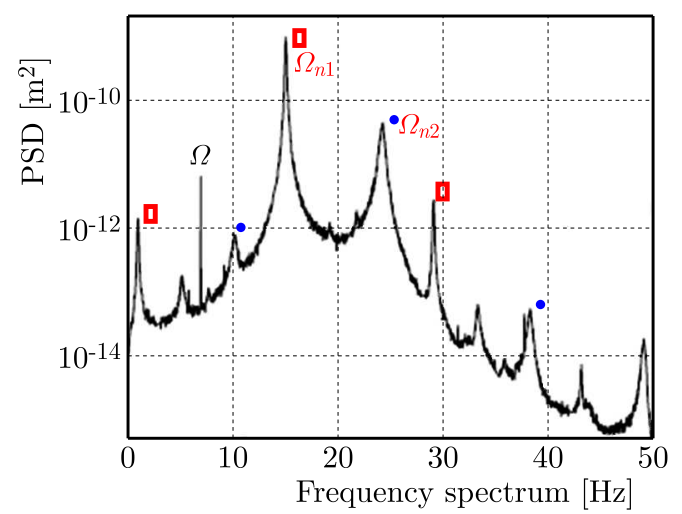

Fig. 12. (a) Frequency response, $C_{0}=1.5 \mathrm{~mm}, I_{0}=3 \mathrm{~A}, A=200 \mathrm{~mm}^{2}$. (b) Experimental frequency response, Lazarus et al. (2010)

The frequency response is essentially characterized by:

- A dominant peak at the rotational frequency $(\Omega=7 \mathrm{~Hz})$ indicating the presence of an unbalance.

- Several peaks located at frequencies of odd multiples of the rotational frequency $(3 \Omega, 5 \Omega, 7 \Omega, \ldots)$ indicating the simultaneous presence of an unbalance and shaft asymmetry as well as representing the modulation phenomenon. Indeed, the mass and stiffness terms are time variables having the frequency equal to two times of that of rotation, whereas the excitation frequency (unbalance force) being equal to the rotational frequency.

- Two peaks presenting the first and second natural frequencies of the rotor (in $x z$-plane): $\Omega_{n 1}=59 \mathrm{~Hz}$ and $\Omega_{n 2}=203 \mathrm{~Hz}$. Supplementary harmonics emerge in the frequency response and are located following the relation: $\Omega_{j}=\Omega_{n i} \pm 2 j \Omega(j>1, i=1,2)$. The fundamental and first secondary harmonics related to the natural frequencies $F_{n 1}$ and $F_{n 2}$ are respectively marked with spots and square marks. These peaks correspond to parametric quasi-modes characterizing every linear time-varying system like an asymmetric shaft. These results are in good agreement with the experimental results given in Fig. 12b and found by Lazarus et al. (2010). Indeed, the authors measured the frequency response of 
an asymmetrical shaft. These experimental results are filtered to remove the spin speed subharmonics. The fundamental and first secondary harmonics related to the natural frequencies $\Omega_{n 1}=15 \mathrm{~Hz}$ and $\Omega_{n 2}=23 \mathrm{~Hz}$ are, respectively, marked with red square marks and with blues pots.

We deliberately chose a low speed rotation (although magnetic bearings operate at high speeds) to validate the numerical simulations. Finally, we note that the same behavior is observed in the $y z$-plane. The parametric quasi-modes also appear regardless of the running speed used.

\section{Conclusion}

In this paper, a finite element procedure for rotor-AMBs systems is generalized to include the effects of shaft asymmetry.

Firstly a model describing electromagnetic bearings (with four electromagnets) has been developed allowing to calculation of the dynamic coefficients which are mainly influenced by the air gap $C_{0}$ between the stator and the shaft, the effective cross-sectional area $A$ and the bias current $I_{0}$. Then, analysis of the stability by Floquet's theory shows that the stability is strongly dependent on the parameters of the bearings (which allow determination of the optimum parameters providing better behavior). The stability of the system is improved by the choice of AMBs parameters leading to an increase in the damping coefficients.

The dynamic response identifies the asymmetry of the shaft by the presence of odd harmonics of the rotational frequency and parametric quasi-modes in frequency spectra. These features could be useful in the detection of shaft faults in diagnosis of rotating machines.

\section{References}

1. Attia Hili M., Fakhfakh T., Haddar M., 2006, Failure analysis of a misaligned and unbalanced flexible rotor, Journal of Failure Analysis and Prevention, 6, 4, 73-82

2. Batoz J.L., Gouri D., 1990, Modelisation des structures par élements finis, Paris Editions Hermès

3. Bauchau O.A., Nikishkov Y.G., 2001, An implicit Floquet analysis for rotorcraft stability evaluation, Journal of The American Helicopter Society, 46, 200-209

4. Bouaziz A., Barkallah M., Bouaziz S., Choley J.-Y., Haddar M., 2016, Cutting parameters and vibrations analysis of magnetic bearing spindle in milling process, Journal of Theoretical and Applied Mechanics, 54, 3, 691-703

5. Bouaziz S., Belhadj Messaoud N., Mataar M., Fakhfakh T., Haddar M., 2011, A theoretical model for analyzing the dynamic behaviour of spatial misaligned rotor with active magnetic bearings, Mechatronics, 21, 899-907

6. Dufour R., Berlioz A., 1998, Parametric instability of a beam due to axial excitations and boundary conditions, ASME Journal of Vibration and Acoustics, 120, 461-467

7. Gosiewski Z., 2008, Control-oriented modeling and control of rotor vibration, Acta Mechanica et Automatica, 2, 2, 21-38

8. InAgaki T., Kanki H., Shiraki K., 1980, Response analysis of a general asymmetric rotorbearing system, Journal of Mechanical Design, 102, 147-157

9. Inayat-Hussain J.I., 2007, Chaos via torus breakdown in the vibration response of a rigid rotor supported by active magnetic bearings, Chaos, Solitions and Fractals, 31, 4, 912-927

10. InAYAT-Hussain J.I., 2010, Nonlinear dynamics of a magnetically supported rigid rotor in auxiliary bearings, Mechanism and Machine Theory, 45, 11, 1651-1667 
11. Lazarus A., Prabel B., Combescure D., 2010, A 3D finite element model for the vibration analysis of asymmetric rotating machines, Journal of Sound and Vibration, 329, 3780-3797

12. Lei S.G., Palazzolo A., 2008, Control of flexible rotor systems with active magnetic bearings, Journal of Sound and Vibration, 314, 19-38

13. Nelson H.D., MCVaugh J.M., 1976, The dynamics of rotor-bearing systems using finite elements, Journal of Engineering for Industry, 98, 593-600

14. Oncescu F., Lakis A.A., Ostiguy G., 2001, Investigation of the stability and steady state response of asymmetric rotors using finite element formulation, Journal of Sound and Vibration, 245, 2, 303-328

15. Tsai N.-C., Li H.-Y., Lin C.-C., Chiang C.-W., Wang P.-L., 2011, Identification of rod dynamics under influence of active magnetic bearing, Mechatronics, 21, 6, 1013-1024

Manuscript received August 24, 2016; accepted for print January 5, 2017 